\title{
Normal state bottleneck and nematic fluctuations from femtosecond quasi-particle relaxation dynamics in $\mathrm{Sm}(\mathrm{Fe}, \mathrm{Co}) \mathrm{AsO}$
}

\author{
T. Mertelj ${ }^{1}$, L. Stojchevska ${ }^{1}$, N. D. Zhigadlo ${ }^{2}$, J. Karpinski ${ }^{2,3}$ and D. Mihailovic ${ }^{1}$ \\ ${ }^{1}$ Complex Matter Dept., Jozef Stefan Institute, Jamova 39, Ljubljana, SI-1000, Ljubljana, Slovenia \\ ${ }^{2}$ Laboratory for Solid State Physics, ETH Zürich, 8093 Zürich, Switzerland and \\ ${ }^{3}$ Institute of Condensed Matter Physics, EPFL, CH-1015 Lausanne, Switzerland
}

(Dated: July 3, 2018)

\begin{abstract}
We investigate temperature and fluence dependent dynamics of the photoexcited quasi-particle relaxation and low-energy electronic structure in electron-doped 1111-structure $\mathrm{Sm}\left(\mathrm{Fe}_{0.93} \mathrm{Co}_{0.07}\right) \mathrm{AsO}$ single crystal. We find that the behavior is qualitatively identical to the 122 -structure $\mathrm{Ba}(\mathrm{Fe}, \mathrm{Co})_{2} \mathrm{As}_{2}$ including the presence of a normal state pseudogap and a marked 2-fold symmetry breaking in the tetragonal phase that we relate to the electronic nematicity. The 2 -fold symmetry breaking appears to be a general feature of the electron doped iron pnictides.
\end{abstract}

\section{INTRODUCTION}

Recently, the presence of the normal-state electronic nematic fluctuations ${ }^{-10}$ in layered iron-based pnictide superconductors 11 has been suggested from a remarkable 2 -fold anisotropy of physical properties in the strained tetragonal phase, well above the structural phase transition. The microscopic origin of the observed anisotropy and possible relation to spin-density-wave (SDW) ordering and superconductivity is still under intense debate.

Most of the published experimental work on 2-fold normal state planar anisotropy is based on 122-structure systems such as Co-doped $\mathrm{BaFe}_{2} \mathrm{As}_{2}$ (Ba-122) with only a few exceptions $\frac{8-10}{\underline{n}}$ Moreover, hole doped $\mathrm{BaFe}_{2} \mathrm{As}_{2}{ }^{5}$ shows only a tiny anisotropy in resistivity indicating that the domain of the presence of the nematicity might be limited to the electron doped compounds. It is therefore important to establish experimentally to what extent the tendency for a 2-fold symmetry breaking is general also for different members of the electron doped iron-based pnictide superconductors family.

Photoexcited carrier dynamics in iron-based pnictides $12-21$ was proven to be a useful probe of the electronic structure. Recently, we demonstrated a remarkable sensitivity of the photoinduced reflectivity transients to the 2-fold symmetry breaking in Co doped Ba-122 $\stackrel{?}{\underline{7}}$ Here we extend our study to Co doped 1111structure $\mathrm{SmFeAsO}(\mathrm{Sm}-1111)$ and find that behavior is qualitatively identical to the behavior in Ba-122, with the clear presence of the 2 -fold anisotropy up to $\sim 170$ $\mathrm{K}$.

\section{EXPERIMENTAL}

\section{A. Setup and sample}

The $\mathrm{SmFe}_{0.93} \mathrm{Co}_{0.07} \mathrm{AsO}$ single crystal with $T_{\mathrm{c}}=15.2$ $\mathrm{K}\left(T_{\mathrm{c} \text {,midpoint }}=14 \mathrm{~K}\right)$ and several hundred micrometers in size was grown from NaAs flux at ETH Zurich using high-pressure and high-temperature technique ${ }^{22}$ For optical measurements the crystal was glued onto a sapphire substrate and cleaved by a razor blade before mounting in an optical liquid-He flow cryostat.

Measurements of the photoinduced reflectivity, $\Delta R / R$, were performed using the standard pump-probe technique, with 50 fs optical pulses from a $250-\mathrm{kHz}$ Ti: $\mathrm{Al}_{2} \mathrm{O}_{3}$ regenerative amplifier seeded with an $\mathrm{Ti}: \mathrm{Al}_{2} \mathrm{O}_{3}$ oscillator. We used the pump photons with the doubled $\left(\hbar \omega_{\mathrm{P}}=3.1\right.$ $\mathrm{eV}$ ) photon energy and the probe photons with the laser fundamental $1.55 \mathrm{eV}$ photon energy to easily suppress the scattered pump photons by long-pass filtering. The pump and probe beams were nearly perpendicular to the cleaved sample surface with polarizations perpendicular to each other and oriented with respect to the crystals to obtain the maximum/minimum amplitude of $\Delta R / R$ at low temperatures. The pump and probe beam diameters at the sample position were $110 \mu \mathrm{m}$ and $52 \mu \mathrm{m}$, respectively.

\section{B. Overview of the experimental data set}

In Fig. 1 we plot the temperature dependence of the raw photoinduced reflectivity $(\Delta R / R)$. In spite of the fact, that no deliberate uniaxial strain was applied to the sample, the transients develop a 2-fold rotational anisotropy with respect to the probe polarization upon cooling. At the room temperature the transients are nearly identical for both polarizations. Below $\sim 200$ $\mathrm{K}$ the transients for different probe polarizations start to show different time dependencies. The transients for one of the polarizations eventually change the sign with decreasing temperature. In the absence of information which crystallographic directions correspond to the two different orthogonal probe polarizations we denote the polarization corresponding to the low-temperature minimal and maximal sub-picosecond peak $\Delta R / R$ value $\mathcal{P}^{-}$ and $\mathcal{P}^{+}$, respectively.

At low temperatures the $\Delta R / R$ transients show a strong pump fluence $(\mathcal{F})$ dependence. In the superconducting state, at the lowest experimentally feasible fluence, the transients in both probe polarizations change sign after the initial sub-ps peak followed by a slow decay 


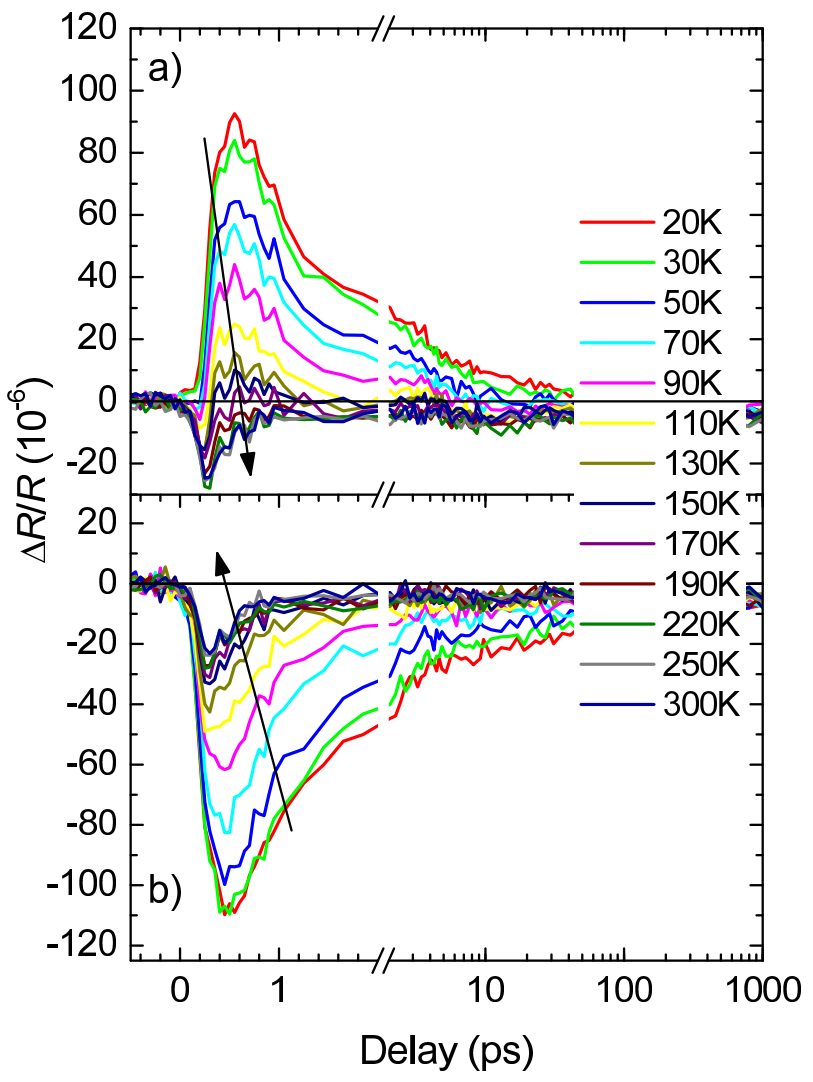

Figure 1. (Color online) The raw $\Delta R / R$ transients as a function of temperature at $8 \mu \mathrm{J} / \mathrm{cm}^{2}$ pump fluence. a) and b) correspond to $\mathcal{P}^{+}$and $\mathcal{P}^{-}$polarizations, respectively. The arrows indicate increasing temperature.

on several-hundred-ps timescale (see Fig. 22). The amplitude of the slow part of the transients shows saturation with increasing $\mathcal{F}$ already at the lowest $\mathcal{F}=0.8 \mu \mathrm{J} / \mathrm{cm}^{2}$, while the sub-ps peak magnitude is linear with $\mathcal{F}$ up to $\mathcal{F} \simeq 10 \mu \mathrm{J} / \mathrm{cm}^{2}$. With further increase of $\mathcal{F}$ the amplitude of the sub-ps peak also saturates and the signal evolves towards the shape observed at the room temperature. At the room temperature the transients show a linear increase of the magnitude with increasing $\mathcal{F}$ with no signs of any saturation or change in the relaxation time.

\section{DISCUSSION}

\section{A. Relaxation components in the normal state}

In order to determine $T$-dependencies of relaxation times we fit the transients for both probe polarizations up to 100 ps delay with three exponentially decaying components: $\underline{23}$

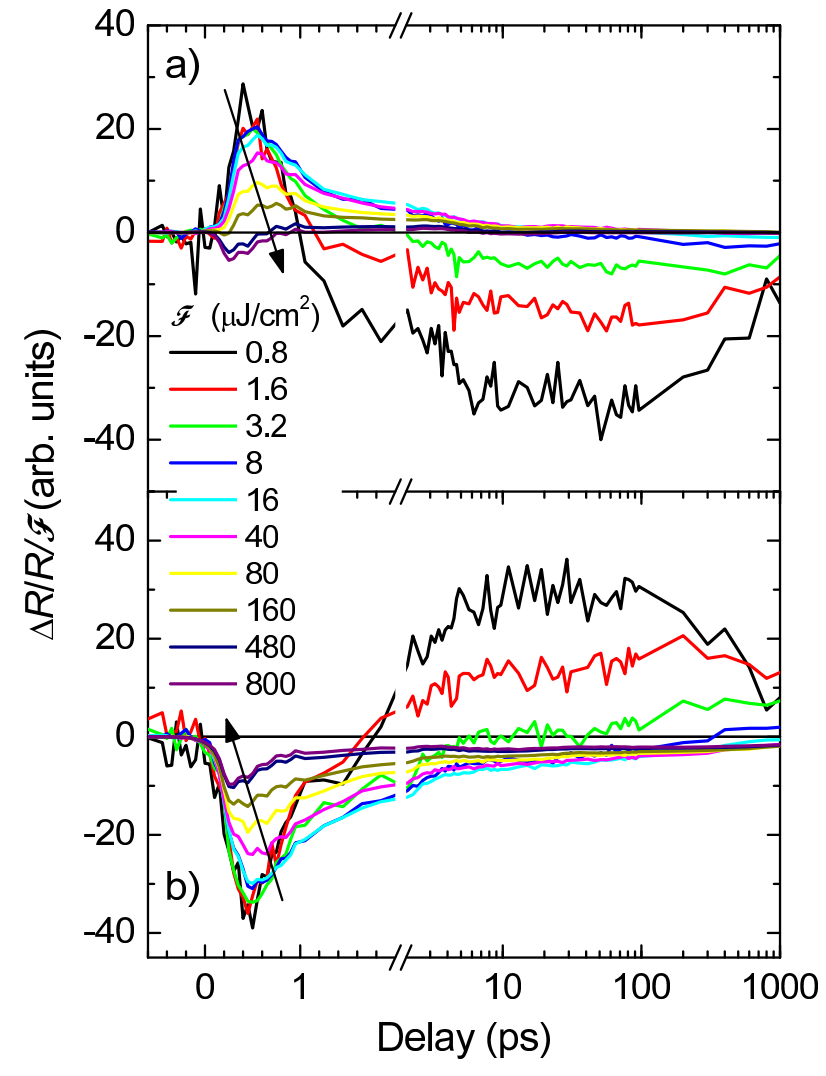

Figure 2. (Color online) Fluence-normalized $\Delta R / R / \mathcal{F}$ transients as a function of $\mathcal{F}$ at $T=5 \mathrm{~K}$. The arrows indicate the direction of increasing $\mathcal{F}$. Overlap of the curves indicates a linear $\mathcal{F}$ dependence. a) and b) correspond to the $\mathcal{P}^{+}$and $\mathcal{P}^{-}$polarization, respectively.

$$
\begin{aligned}
\frac{\Delta R}{R}= & \sum_{i \in\{\mathrm{A}, \mathrm{B}, \mathrm{C}\}} \frac{A_{i}}{2} \mathrm{e}^{-\frac{t-t_{0}}{\tau_{i}}} \operatorname{erfc}\left(\frac{\sigma^{2}-4\left(t-t_{0}\right) \tau_{i}}{2 \sqrt{2} \sigma \tau_{i}}\right)+ \\
& +\frac{A_{\mathrm{D}}}{2} \operatorname{erfc}\left(-\frac{\sqrt{2}\left(t-t_{0}\right)}{\sigma}\right)
\end{aligned}
$$

where $\sigma$ corresponds to the effective width of the excitation pulse with a Gaussian temporal profile arriving at $t_{0}$ and $\tau_{i}$ the exponential relaxation times. Component $\mathrm{D}$ represents the relaxation on a timescale beyond 100 ps. During the fitting the relaxation times for the two orthogonal polarizations were linked while amplitudes were kept independent. Examples of fits are shown in Fig. 3 for the three characteristic temperature regions.

There is a systematic discrepancy between the fits and the data for $\mathcal{P}^{+}$indicating the presence of another weak component. The fit error shown in the inset to Fig. 3 suggest that this component is rather weak and temperature independent so it will not be discussed further here.

At the highest temperatures a single exponential component named A, with a linearly $T$-dependent relaxation time, $\tau_{\mathrm{A}} \sim 0.2-0.3 \mathrm{ps}$, completely describes the initial sub-ps relaxation (see Fig. 3 and (4). 

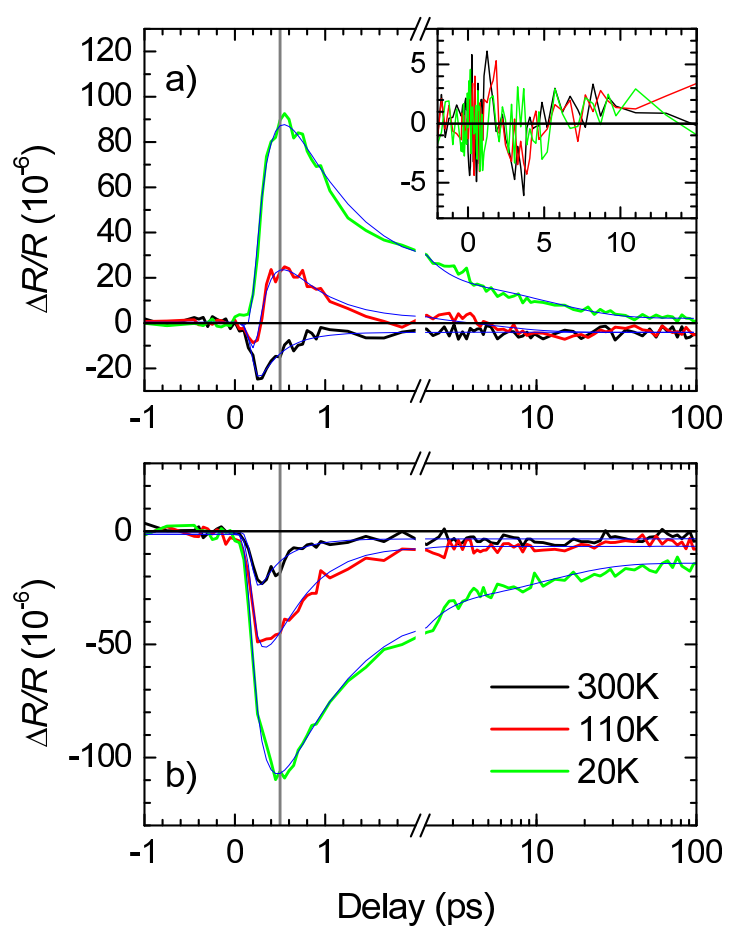

Figure 3. (Color online) Examples of multi-exponent fits (1) in the normal state for both polarizations. The inset shows the systematic fit error observed for the $\mathcal{P}^{+}$polarization. The vertical lines represent the peaks of the low- $T$ transients at $0.5 \mathrm{ps}$.

Below $\sim 170 \mathrm{~K}$, where the anisotropy becomes clearly visible and the $\mathcal{P}^{+}$- polarization component A changes sign, another component named B appears with a slightly longer relaxation time and a strong amplitude anisotropy. The relaxation time, $\tau_{\mathrm{B}}$, [see Fig. 4(a)] shows an increase with decreasing $T$ from $\sim 0.4$ ps at $\sim 170 \mathrm{~K}$ to $\sim 0.7 \mathrm{ps}$ at $5 \mathrm{~K}$, while $\tau_{\mathrm{A}}$ remains constant slightly below $0.2 \mathrm{ps}$ in this $T$-range.

Component $\mathrm{C}$ [see Fig. 5] has the longest relaxation time $\left(\tau_{\mathrm{C}}\right)$. The component is absent at the room $T$ and appears below $\sim 250 \mathrm{~K}$. The amplitude is rather small an $T$-independent down to $\sim 120 \mathrm{~K}$ and then increases with decreasing temperature and saturates below $\sim 70$ K. $\tau_{\mathrm{C}}$ is, differently from the amplitude, temperature independent above $\sim 70 \mathrm{~K}$ while it steeply increases with decreasing $T$ in the region where the amplitude saturates.

The amplitude of component D is also anisotropic at the lowest $T$. With increasing $T$ the anisotropy decreases and vanishes above $120 \mathrm{~K}$, were the amplitude becomes $T$-independent.

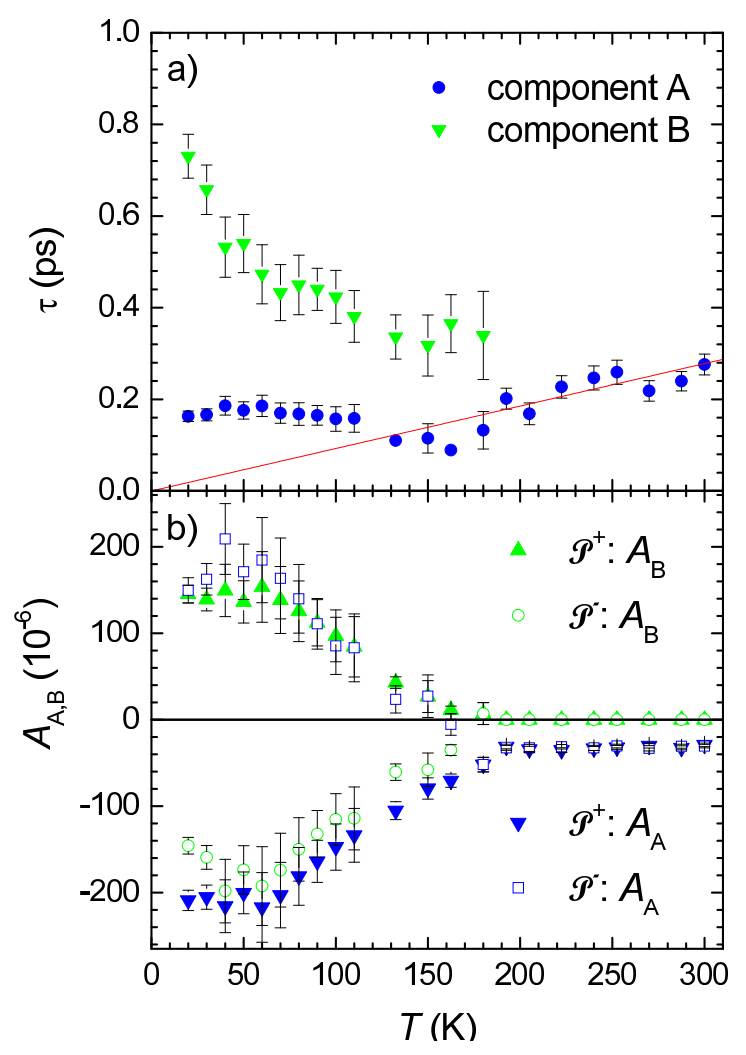

Figure 4. (Color online) The fastest two multi-exponential fit relaxation times a) and corresponding amplitudes b). The red (gray) line is a fit of Eq. (3) discussed in the text.

\section{B. Pseudogap and 2-fold in-plane anisotropy}

Above $T_{\mathrm{c}}$ the amplitudes of the transients show a gradual decrease with increasing temperature (see Fig. 6). Such a decrease can be associated with a bottleneck in relaxation and therefore the presence of a pseudogap in the quasiparticle density of states. To obtain a quantitative information about the pseudogap we analyze the normal state $T$-dependent amplitudes in the context of the relaxation across a $T$-independent gap. $12,14,24$ :

$$
\Delta R \propto n_{\mathrm{pe}} \propto\left[1+g_{\mathrm{ph}} \exp \left(-\frac{\Delta_{\mathrm{PG}}(T)}{k_{\mathrm{B}} T}\right)\right]^{-1},
$$

where $g_{\mathrm{ph}}$ is the ratio between the number of involved phonons and number of involved quasi-particle states. 24 As shown in Fig. 6] the T-dependent amplitude for the $\mathcal{P}^{-}$polarization can be well fit using equation (2) with $2 \Delta_{\mathrm{PG}}=440 \mathrm{~K}$. This value is slightly lower than in Co doped Ba-122?

On the other hand, the $T$-dependent transients for the $\mathcal{P}^{+}$polarization show the change of sign with increasing $T$ including a non-monotonous relaxation in the 90-170 $K$ range and can not be described directly by equation (2) ${ }^{25}$ The failure of Eq. (2) for description of the $\mathcal{P}^{+}$ 


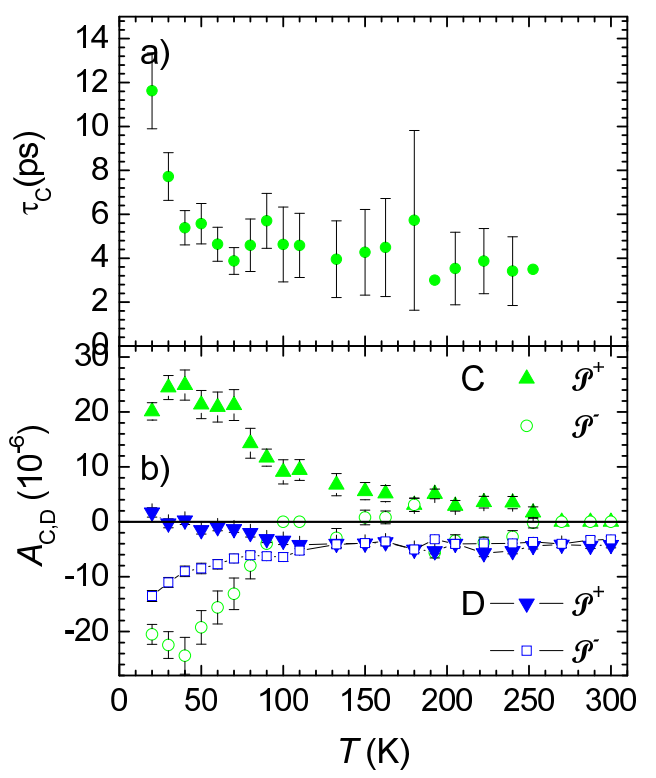

Figure 5. (Color online) The slowest multi-exponential fit relaxation time a) and the corresponding amplitude b) together with the long-delay residual $A_{\mathrm{D}}$.

polarization amplitude $T$-dependence, however, does not contradict to the bottleneck. Eq. (2) does not take into account the intrinsic multi-band nature of iron-pnictides leading to the multi component relaxation and a possible $T$-dependence of the optical-transition matrix elements. Indeed, the $T$-dependent amplitudes of each component separately (Fig. 4 (b)) are consistent with the bottleneck scenario while the conspicuous sign change of $\mathcal{P}^{+}$polarization component A amplitude at $\sim 170 \mathrm{~K}$ can be attributed to the change of the optical matrix elements due to the band shifts as discussed already for the SDW state in Co doped Ba-122 $\stackrel{7}{?}$

Comparing the relaxation times of the strongest two relaxation components with time resolved ARPES 21 in Eu-122 shows a striking similarity in both magnitudes and temperature dependencies. According to ARPES different relaxation times correspond to different regions in the Brillouine zone, where in the SDW state the slower relaxation, with $\tau \sim 0.8 \mathrm{ps}$, is due to the relaxation of the photexcited electrons around the $\Gamma$ point and the fastest, with $\tau \sim 0.15$ ps corresponds to the photexcited holes away from the the $\Gamma$ point. Since our sample shows no SDW ordering the similarity suggests that the pseudogap has a similar origin as the charge gap observed in the SDW state.

As in the case of Co-doped Ba- $122^{7}$ the most striking feature of our data set is the observation of the 2 -fold

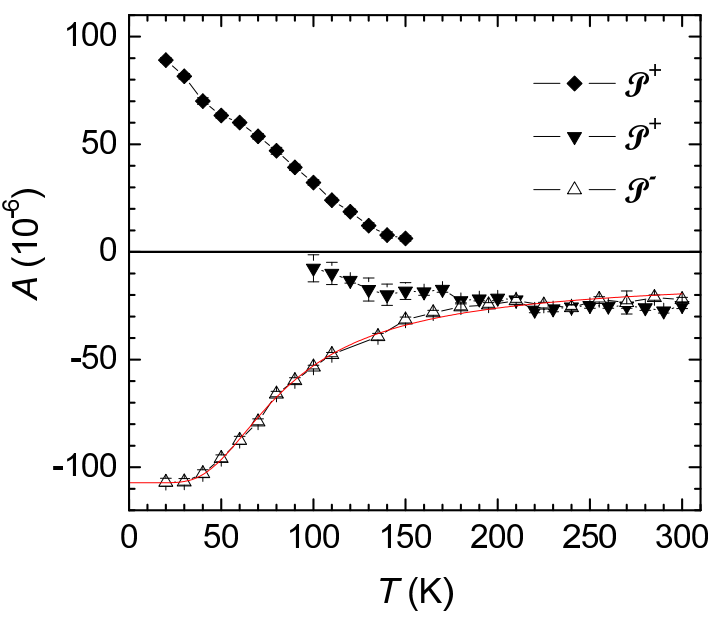

Figure 6. (Color online) The magnitudes of $\Delta R / R$ transients extrema as a function of $T$. The red (gray) lines is the fit of Eq. (2) discussed in the text.

in-plane rotational anisotropy of the optical transients despite the tetragonal lattice symmetry (see Fig. 1). In the absence of any structural data ${ }^{26}$ which would indicate that our sample is not tetragonal in the thermodynamic equilibrium we assume that the breaking of the 4 -fold tetragonal symmetry is not spontaneous, but is a consequence of an external anisotropy such as anisotropic boundary and/or excitation conditions that introduce an anisotropic surface strain $\underline{\underline{7}}$ Since in our experiment any external anisotropy must be weak our observations suggest that the intrinsic susceptibility for 2-fold symmetry breaking is large and increases with decreasing $T$.

Using the same arguments as in the case of Co doped Ba-122 we tentatively associate the 2-fold symmetry breaking instability with electronic nematic fluctuations 1 or ordering of the $\mathrm{Fe} d$ orbitals. Due to the concurrent appearance of the bottleneck and the increase of the polarization anisotropy of the transients during cooling the pseudogap might be associated with the 4 -fold rotational symmetry breaking.

Our observations are consistent with the recently observed ${ }^{9,10}$ weak structural tetragonal symmetry breaking above $T \simeq 140 \mathrm{~K}$ in $\mathrm{F}$ doped $\mathrm{Sm}-1111$ by a high resolution synchrotron powder diffraction, extending well into the SC dome region of the phase diagram.

\section{Electron phonon coupling}

Since our data suggest that the effects of the nematic fluctuations become negligible above $T \simeq 200 \mathrm{~K}$ we analyze the initial part of the $\Delta R / R$ transients at high $T$ in the framework of the electron-phonon relaxation in metals $\underset{27,28}{2}$ The $\mathcal{F}$-independent relaxation at 
the room temperature warrants use of the low excitation expansion,, 27 where in the high temperature limit the energy relaxation time is proportional to $T, \underline{27,28}$

$$
\tau=\frac{2 \pi k_{\mathrm{B}} T}{3 \hbar \lambda\left\langle\omega^{2}\right\rangle} .
$$

Here $\lambda\left\langle\omega^{2}\right\rangle$ is the second moment of the Eliashberg function, $\alpha^{2} F(\omega)$, and $k_{\mathrm{B}}$ the Boltzman constant. 27 .

The temperature dependence of component A relaxation time shows a clear linear $T$-dependence predicted by equation (3) above $T \simeq 200 K$ corresponding to $\lambda\left\langle\omega^{2}\right\rangle=130 \mathrm{meV}^{2}$, which is the same as in the undoped $\mathrm{Sm}-1111^{14}$ and suggests a moderate electron phonon coupling constant $\lambda \sim 0.2$.

\section{Superconducting state}

The slowest part of the relaxation shows a strong temperature and fluence dependencies below $T_{\mathrm{c}}$. Since at low $\mathcal{F}$ there is virtually no $T$-dependence of the transients above $T_{\mathrm{c}}=15.2 \mathrm{~K}$ up to $\sim 30 \mathrm{~K}$ (see Fig. 17) we extract the slow superconducting (SC) component by subtraction of the averaged transients measured just above $T_{\mathrm{c}}$, as shown in Fig. 7 (b). At $\mathcal{F}=3.2 \mu \mathrm{J} / \mathrm{cm}^{2}$ the SC component has a risetime of $\sim 1$ ps and shows significantly slower decay time than our time window. Consistently with previous observations in the cuprates and iron-based pnictides $29-31$ the $\mathrm{SC}$ component is associated with a complete photodestruction of the SC state.

We fit the temperature dependence of the saturated SC-component amplitude (see inset to Fig. 7) using the high-frequency limit of the Mattis-Bardeen formula, $\stackrel{14,32}{,}$

$$
\frac{\Delta R}{R} \propto\left(\frac{\Delta(T)}{\hbar \omega}\right)^{2} \log \left(\frac{3.3 \hbar \omega}{\Delta(T)}\right)
$$

where $\hbar \omega$ is the probe-photon energy and $\Delta(T)$ the superconducting gap with the BCS temperature dependence. From the fit we obtain $T_{\mathrm{c}}=13.3 \mathrm{~K}$ which is 1.9 $\mathrm{K}$ smaller than $T_{\mathrm{c}}$ from the SQUID magnetometry data. Due to the small signal to noise level of the SC component it is not possible to reliably determine whether this discrepancy originates from the laser heating of the excited volume $\mathrm{B}^{33}$ or the sample is inhomogeneous with variations of $T_{\mathrm{c}}$ across the sample as suggested by the width of the susceptibility transition with $T_{\mathrm{c}}-T_{\mathrm{c} \text {,midpint }}=1.2 \mathrm{~K}$.

Moreover, the SC component shows a similar anisotropy as the normal state components. The presence of the anisotropy concurrent with the presence of transients due to the pseudogap bottleneck below $T_{\mathrm{c}}$ indicate that the superconductivity is coexisting with nematicity and the pseudogap.

\section{SUMMARY AND CONCLUSIONS}

We performed a time-resolved optical spectroscopy study in near optimally doped $\mathrm{Sm}\left(\mathrm{Fe}_{0.93} \mathrm{Co}_{0.07}\right) \mathrm{AsO}$
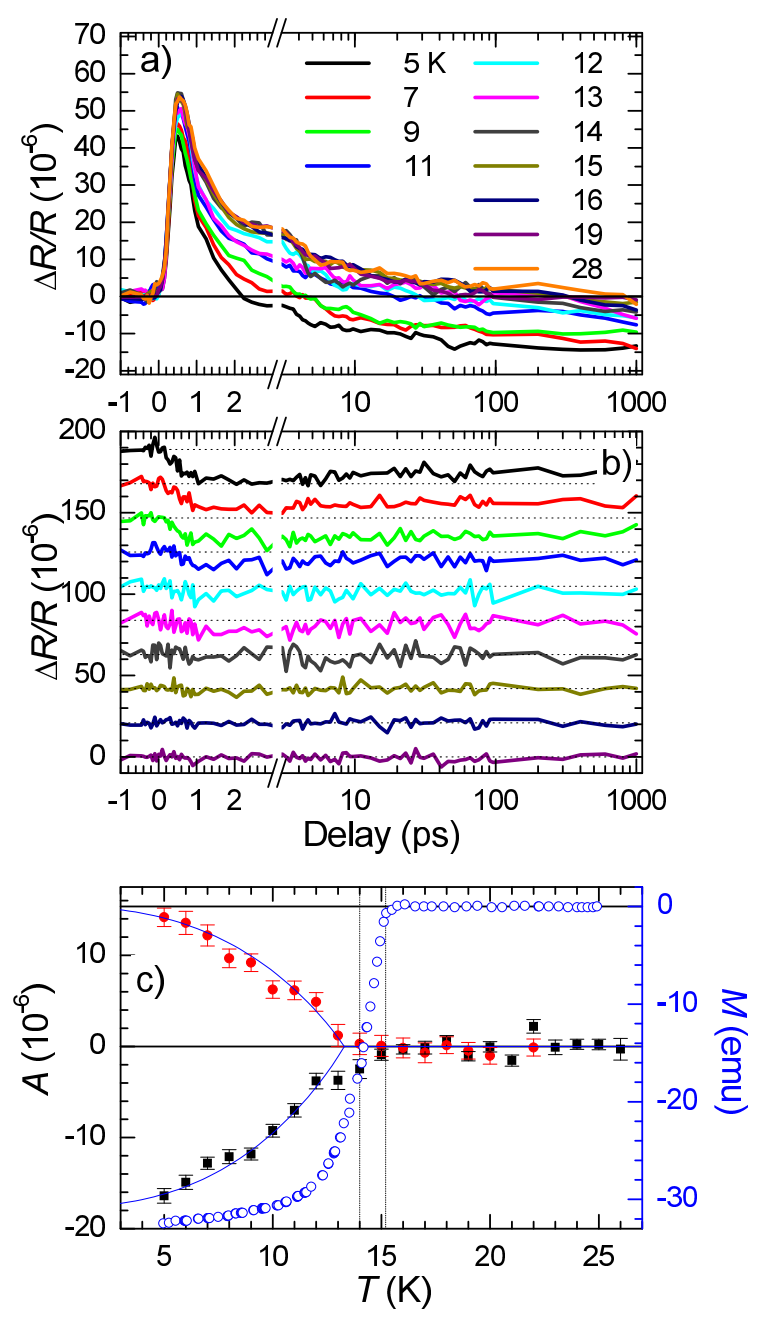

Figure 7. (Color online) The raw $\Delta R / R$ transients as a function of $T$ at $3.2 \mu \mathrm{J} / \mathrm{cm}^{2}$ pump fluence for the $\mathcal{P}^{+}$polarization (a). The superconducting response for the $\mathcal{P}^{+}$polarization obtained by subtraction of the response above $T_{\mathrm{c}}$ (b). The traces are shifted vertically for clarity as indicated by the dotted lines. The magnitude of the SC response for $\mathcal{P}^{+}$and $\mathcal{P}^{-}$polarizations as functions of $T$ compared to the SQUID magnetometry data (c). The lines in (c) are Mattis-Bardeen fits (4) discussed in text. The vertical dashed lines indicate $T_{\mathrm{c}}$ and $T_{\mathrm{c} \text {,midpoint }}$ obtained from the SQUID susceptibility data.

pnictide superconductor. As in all previously studied electron doped iron pnictide superconductors ${ }^{7.14}$ we find a normal state bottleneck in the relaxation associated with opening of a pseudogap in the electronic density of states and a moderate electron phonon coupling constant.

Due to the observation of a marked 2-fold in-plane anisotropy below $\sim 170 \mathrm{~K}$, that is concurrent with the appearance of the pseudogap, we tentatively associate the anomalous normal state properties with electronic nematic fluctuations, similar as found in $\mathrm{Ba}(\mathrm{Fe}, \mathrm{Co})_{2} \mathrm{As}_{2}$. 
The high susceptibility towards 2 -fold symmetry breaking therefore seems to be a general feature of electron doped (Fe,As) planes. The presence of the anisotropy in the superconducting state indicates a coexistence of superconductivity and the nematic electronic order in these compounds.

\section{ACKNOWLEDGMENTS}

Work at Jozef Stefan Institute was supported by ARRS (Grant No. P1-0040). The work at ETH Zürich was supported by Swiss National Science Foundation and the National Center of Competence in Research MaNEP. J. K. acknowledges support from ERC project Super Iron.
1 J.-H. Chu, J. G. Analytis, K. De Greve, P. L. McMahon, Z. Islam, Y. Yamamoto, and I. R. Fisher, Science 329, 824 (2010)

2 T.-M. Chuang, M. P. Allan, J. Lee, Y. Xie, N. Ni, S. L. Bud'ko, G. S. Boebinger, P. C. Canfield, and J. C. Davis, Science 327, 181 (2010)

3 M. A. Tanatar, E. C. Blomberg, A. Kreyssig, M. G. Kim, N. Ni, A. Thaler, S. L. Bud'ko, P. C. Canfield, A. I. Goldman, I. I. Mazin, and R. Prozorov, Phys. Rev. B 81, 184508 (2010).

4 Dusza, A., Lucarelli, A., Pfuner, F., Chu, J.-H., Fisher, I. R., and Degiorgi, L., EPL 93, 37002 (2011)

5 J. J. Ying, X. F. Wang, T. Wu, Z. J. Xiang, R. H. Liu, Y. J. Yan, A. F. Wang, M. Zhang, G. J. Ye, P. Cheng, J. P. Hu, and X. H. Chen, Phys. Rev. Lett. 107, 067001 (2011).

${ }^{6}$ M. Yi, D. Lu, J.-H. Chu, J. G. Analytis, A. P. Sorini, A. F. Kemper, B. Moritz, S.-K. Mo, R. G. Moore, M. Hashimoto, W.-S. Lee, Z. Hussain, T. P. Devereaux, I. R. Fisher, and Z.-X. Shen, Proceedings of the National Academy of Sciences 108, 6878

7 L. Stojchevska, T. Mertelj, J. Chu, I. Fisher, and D. Mihailovic, Physical Review B 86, 024519 (2012).

8 Y. Zhang, C. He, Z. R. Ye, J. Jiang, F. Chen, M. Xu, Q. Q. Ge, B. P. Xie, J. Wei, M. Aeschlimann, X. Y. Cui, M. Shi, J. P. Hu, and D. L. Feng, Phys. Rev. B 85, 085121 (2012)

9 A. Martinelli, A. Palenzona, M. Tropeano, M. Putti, C. Ferdeghini, G. Profeta, and E. Emerich, Phys. Rev. Lett. 106, 227001 (2011).

10 A. Martinelli, A. Palenzona, M. Putti, and C. Ferdeghini, Phys. Rev. B 85, 224534 (2012)

11 Y. Kamihara, T. Watanabe, M. Hirano, H. Hosono, et al., J. Am. Chem. Soc 130, 3296 (2008).

12 T. Mertelj, V. Kabanov, C. Gadermaier, N. Zhigadlo, S. Katrych, J. Karpinski, and D. Mihailovic, Physical Review Letters 102, 117002 (2009).

13 T. Mertelj, V. Kabanov, C. Gadermaier, N. Zhigadlo, S. Katrych, Z. Bukowski, J. Karpinski, and D. Mihailovic, Journal of Superconductivity and Novel Magnetism 22, 575 (2009).

14 T. Mertelj, P. Kusar, V. V. Kabanov, L. Stojchevska, N. D. Zhigadlo, S. Katrych, Z. Bukowski, J. Karpinski, S. Weyeneth, and D. Mihailovic, Phys. Rev. B 81, 224504 (2010).

15 D. H. Torchinsky, G. F. Chen, J. L. Luo, N. L. Wang, and N. Gedik, Phys. Rev. Lett. 105, 027005 (2010)

16 E. E. M. Chia, D. Talbayev, J.-X. Zhu, H. Q. Yuan, T. Park, J. D. Thompson, C. Panagopoulos, G. F. Chen, J. L. Luo, N. L. Wang, and A. J. Taylor, Phys. Rev. Lett. 104, 027003 (2010).

17 L. Stojchevska, P. Kusar, T. Mertelj, V. V. Kabanov, X. Lin, G. H. Cao, Z. A. Xu, and D. Mihailovic,
Phys. Rev. B 82, 012505 (2010).

18 Y. Gong, W. Lai, T. Nosach, L. J. Li, G. H. Cao, Z. A. Xu, and Y. H. Ren, New Journal of Physics 12, 123003 (2010)

19 B. Mansart, D. Boschetto, A. Savoia, F. RullierAlbenque, F. Bouquet, E. Papalazarou, A. Forget, D. Colson, A. Rousse, and M. Marsi, Phys. Rev. B 82, 024513 (2010).

20 D. H. Torchinsky, J. W. McIver, D. Hsieh, G. F. Chen, J. L. Luo, N. L. Wang, and N. Gedik, Phys. Rev. B 84, 104518 (2011).

21 L. Rettig, R. Cortés, S. Thirupathaiah, P. Gegenwart, H. S. Jeevan, M. Wolf, J. Fink, and U. Bovensiepen, Phys. Rev. Lett. 108, 097002 (2012).

22 N. D. Zhigadlo, S. Weyeneth, S. Katrych, P. J. W. Moll, K. Rogacki, S. Bosma, R. Puzniak, J. Karpinski, and B. Batlogg, Phys. Rev. B 86, 214509 (2012)

23 D. Mihailovic and J. Demsar, "Spectroscopy of Superconducting Materials," (American Chemical Society: Washington, DC, 1999) Chap. Time-resolved optical studies of (201 quasiparticle dynamics in high-temperature superconductors, pp. 230-244.

${ }^{24}$ V. V. Kabanov, J. Demsar, B. Podobnik, and D. Mihailovic, Phys. Rev. B 59, 1497 (1999).

25 The behavior is similar to the Co doped Ba-122, $\underset{7}{=}$ but the roles of polarizations are reversed presumably due to slightly different optical matrix elements.

26 A. Khan, A. Rahman, S. Naher, H. Kaneko, Y. Yun, A. Savinkov, H. Suzuki, Y. Li, Q. Tao, G. Cao, et al., Journal of Scientific Research 4, 541 (2012).

27 V. V. Kabanov and A. S. Alexandrov, Physical Review B (Condensed Matter and Materials Physics) 78, 174

28 C. Gadermaier, A. S. Alexandrov, V. V. Kabanov, P. Kusar, T. Mertelj, X. Yao, C. Manzoni, D. Brida, G. Cerullo, and D. Mihailovic, Phys. Rev. Lett. 105, 257001 (2010).

29 P. Kusar, V. Kabanov, J. Demsar, T. Mertelj, S. Sugai, and D. Mihailovic, Physical Review Letters 101, 227001 (2008).

30 L. Stojchevska, P. Kusar, T. Mertelj, V. V. Kabanov, Y. Toda, X. Yao, and D. Mihailovic, Phys. Rev. B 84, 180507 (2011).

31 G. Coslovich, C. Giannetti, F. Cilento, S. Dal Conte, G. Ferrini, P. Galinetto, M. Greven, H. Eisaki, M. Raichle, R. Liang, A. Damascelli, and F. Parmigiani, Phys. Rev. B 83, 064519 (2011).

32 D. C. Mattis and J. Bardeen, Phys. Rev. 111, 412 (1958)

33 Due to the laser heating the temperature of the excited spot on the sample is higher than the cryostat temperature leading to an apparent decrease of $T_{\mathrm{c}}$. 